\title{
Application of EMC in Electronic Instrument PCB Design
}

Zhu LiBo

Tianjin Global Magnetic Card Co., Ltd. Tianjin 300202

Abstract: With the rapid development of high-speed integrated circuit technology and computer technology, not only has the application field of electronic products been expanded, but also brought about the complicated system, the mutual interference between various components or systems, the basic part of electronic products Is based on the PCB board to complete the various functions of the functional modules in the electronic product development process, the rapid evaluation of PCB board function module of the electromagnetic interference problem is to shorten the electronic product design cycle, improve product reliability and effective means. This paper is in this context, the design of a set of PCB board EMC problem automatic detection device to quickly, intuitively and accurately find possible sources of electromagnetic interference for electronic product design engineers to provide low-cost, fast testing method.

Key words: electronic equipment; PCB design; EMC

\section{Introduction}

High-speed PCB design continues to be a new challenge compared to traditional PCB-printed board designs, and high-speed PCB design technology has become a hot research area and continues to evolve. In recent years, some studies have shown that high-speed PCB designers should correct or abandon some of the traditional understanding and practice. In this paper, from the application point of view, combined with high-speed PCB design technology in recent years, some research results to explore the current high-speed PCB design in a number of errors and countermeasures ${ }^{1}$.

\section{EMC Overview}

Electromagnetic compatibility EMC is a new comprehensive discipline. The main research is how to make the same electromagnetic environment in the work of a variety of electrical and electronic equipment and components can work properly, do not interfere with each other to achieve electromagnetic compatibility ${ }^{2}$ EMC is a rapidly developing interdisciplinary, the theoretical basis of the design includes electromagnetic field and electromagnetic wave theory, antenna and radio wave propagation, circuit theory, and many other areas of technology include electronic information, communications, radio and television, computer and information equipment, aviation aerospace, transportation, medical equipment, household appliances and so on. Here are some important concepts in the field of electromagnetic compatibility and EMC test content and standards, as well as EMC certification and elaborate EMC for the importance of the product. EMC testing generally consists of two parts, the first part is the test of interference emissions and the other is the sensitivity of the test. Interference test inside the test there are a number of test content, sensitivity test which also has a lot of test content.

\section{System design}

\subsection{Hardware control circuit design of lower computer}

In the design of PCB board EMC automatic test system, the lower computer needs to complete the work there are two main aspects: (1) Communication with the host computer to accept orders from the host computer, the next bit hardware circuit in accordance with the host computer command control two-dimensional movement of the arm movement, to achieve the PCB scan. (2) The control of the mechanical two-dimensional moving arm, that is, the control of the stepper motor. Based on the cost and 
ease of use and maintenance and adapt to the needs of the system, the hardware control circuit selects a low power, high performance and low cost STC89C52 chip produced by STC as a control chip ${ }^{3}$. The chip has a small size, strong function, wide application, low cost and many other advantages. The overall design of the hardware circuit under the machine as shown in Figure 1. The main function of the single-chip central processing unit is to communicate with the host computer, from the host computer to get the implementation of instructions, and then single-chip control $\mathrm{X} / \mathrm{Y}$ direction of the stepper motor movement, while the use of limit sensors on the stepper motor closed-loop control, When the sensor signal responds, the microcontroller stops the stepping motor and improves the stability of the two-dimensional motion platform.

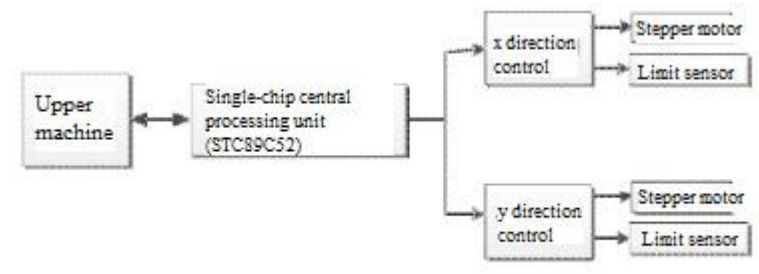

Figure 1 Lower machine hardware circuit overall design block diagram

\subsection{PC software design}

The host computer software is an important part of the design automatic test system. It not only needs to communicate with the lower computer to control the movement of the two-dimensional movement arm. While also controlling the spectrum analyzer, real-time communication with the spectrum analyzer, from the spectrum analyzer to read the measurement data $^{4}$. When the test is completed, the host computer software also need to collect the data analysis, given the test results report. So the host computer software as a central, so that the entire test process in an orderly manner. In summary, from the functional division of the host computer software to complete the three functions. 1) Remote control of the spectrum analyzer 2) Communication with the lower computer to control the movement of the two-dimensional movement of the arm 3) Data processing module so this chapter above the machine software function as the main line, the host computer software in detail. So as to get the integrity of the host computer software architecture.

The display of the measurement data is an indispensable part of the automatic measurement system, and the user does not have to study the specific measurement data and visually see the image drawn by the data, thus greatly reducing the data analysis time. In VB to achieve the data display can be divided into two methods: One is to use VB language to write graphics, the other method is to use graphical display controls ${ }^{5}$. These graphical display controls such as the CWGraph3D control in the Measurement stdio software, the graph's control for the office, and so on. Comparison of these two methods The first method of writing with VB language graphics display not only complex procedures but also there are many difficult to solve problems such as coordinate problems, program maintenance costs and so low efficiency and low display at the same time the effect is not good. In order to facilitate programming at the same time to obtain a good display and consider the complexity of the program and maintainability, the program uses a second graphical display method that uses a graphical display control and use CWGraph control and CWGraph3D control, CWGraph control used to display in a A curve of the data read from the spectrum at the measurement position, which is also the measurement curve that is displayed when the lower computer displays the spectrum at the measurement position,

\subsection{Design of Planar Magnetic Field Detection Antenna}

In this design, the design of the magnetic field detection antenna is planarized and fed in the form of a coplanar waveguide. This structure of the antenna not only to meet the needs of testing, easy to test the installation, and has a very good application value. At the same time the use of coplanar waveguide form of the antenna in the antenna design is also an important research direction. The main antenna theory used in the planar magnetic field detection antenna has two main aspects: the first is the transmission line theory and the second is the asymmetric coplanar waveguide transmission line theory. The magnetic field detection antenna mainly measures the changing magnetic field generated by the high frequency current. Used to diagnose radiation from components, printed circuit boards, integrated circuits, cables, electromagnetic leaks on shielded enclosures, and similar electromagnetic interference. The measured electromagnetic interference signal can be displayed on the spectrum analyzer. With the magnetic field detection antenna can distinguish printed circuit boards, cables and electronic components on the "hot spots" to find out the radiation of large components or 
circuit location. This is useful for finding and removing electromagnetic interference.

In summary, the automated test is the development of today's test technology, this issue combined with PCB board EMC testing proposed a set of automatic test system, the test system is not only on the traditional PCB board EMC testing innovation, while giving future EMC the test which presents a new idea of saving manpower, material and financial resources. The test results show that the test system can effectively measure the electromagnetic interference on the PCB board, can effectively help save the circuit board design cycle, improve work efficiency, can for enterprises, research institutions, etc. to bring convenience and potential profits.

\section{References}

[1] Chung, S., Heo, G., Kwak, J., Oh, S., Lee, Y., Kang, C. and Lee, T., 2013, May. Development of PCB design guide and PCB deformation simulation tool for slim PCB quality and reliability. In Electronic Components and Technology Conference (ECTC), 2013 IEEE 63rd (pp. 2157-2162). IEEE
[2] Gong Chenglong, Han Xiaochun, Chen Jiachen. Application of EMC Technology in PCB Design of Electronic Equipment $[\mathrm{J}]$. Electrical Measurement and Instrumentation, 2005, 05: 32-36.

[3] Wang Xiujie, Zhou Shenghai. Some Errors and Countermeasures High-Speed PCB Design [J]. Journal of Xinyang Normal University (Natural Science Edition), 2007, 01: 120-123.

[4] Marinescu, A., Coatu, S. and Rucinschi, D., 2012, October. About the EMC of non-conventional electronic instrument transformer case study. In Applied and Theoretical Electricity (ICATE), 2012 International Conference on (pp. 1-5). IEEE.

[5] Application of EMC in PCB Design of Electronic Instrument $[\mathrm{J}]$. Information Recording Materials, 2017,18(07):76-77. [2017-09-22]. DOI : 10.16009/j.cnki.cn13-1295/tq.2017.07.045. 